\title{
Emerging strategies for combination checkpoint modulators in cancer immunotherapy
}

\author{
Aleksandra Popovic, Elizabeth M. Jaffee, and Neeha Zaidi \\ The Sidney Kimmel Comprehensive Cancer Center, The Skip Viragh Center for Pancreatic Cancer, The Bloomberg-Kimmel Institute for Cancer Immunotherapy, The Johns Hopkins University \\ School of Medicine, Baltimore, Maryland, USA.
}

\begin{abstract}
Current immune checkpoint-modulating agents have demonstrated clinical efficacy in certain tumor types, particularly those with a high burden of tumor-specific neoantigens, high tumor-mutational burden, and abundant tumor-infiltrating T cells. However, these tumors often stop responding, with signs of T cells exhaustion, decreased T cell effector function, and upregulated inhibitory checkpoints. To enhance antitumor immunity and rescue exhausted T cells, newer inhibitory and stimulatory checkpoint modulators are being tested as monotherapy or in combination with approved checkpoint inhibitors. In contrast, tumors with low tumor-mutational burden, low neoantigen burden, and a paucity of T cells are immunologically "cold," and therefore first require the addition of agents to facilitate the induction of T cells into tumors. Cold tumors also often recruit immunosuppressive cell subsets, including regulatory T cells, myeloid-derived suppressor cells, and macrophages, and secrete immunosuppressive soluble cytokines, chemokines, and metabolites. To unleash an optimal antitumor immune response, combinatorial therapeutics that combine immune checkpoints with other modalities, such as vaccines, are being developed. From current preclinical data, it appears that combinatorial strategies will provide robust and durable responses in patients with immunologically cold cancers.
\end{abstract}

\section{Introduction}

Harnessing the immune system to fight malignancies has become a major focus in cancer therapy. The idea was first introduced in the early 1900s by William Cooley, who attempted to treat sarcoma by provoking the immune system with heat-inactivated bacterial toxins (1). This approach was followed decades later by the concepts of immunosurveillance and immunoediting, which highlighted the monitoring and elimination, respectively, of cancer cells as functional roles of the immune system. It also became clear that tumors escape from immune attack due to the emergence of variant clones (2-4) arising from genomic and epigenetic modifications, including nonsynonymous mutations encoding foreign antigens (i.e., neoantigens) that arise during tumorigenesis (5).

Major histocompatibility complex (MHC) molecules located on antigen-presenting cells (APCs), such as dendritic cells, present foreign tumor antigens to $\mathrm{T}$ cells in the lymph nodes. $\mathrm{T}$ cell priming and activation occur when the MHC-peptide complex interacts with the T cell receptor (TCR), followed by the engagement of CD28 to B7.1 (CD80) or B7.2 (CD86) $(4,5)$. This activation is regulated by both stimulatory and inhibitory checkpoints, a balance that maintains self-tolerance and prevents autoimmunity. Effector T cells (Teffs) then traffic to and interact with tumor cells that present cognate antigens on MHC molecules. These T cells, however, are also subject to the upregulation of inhibitory

Conflict of interest: Under a licensing agreement between Aduro Biotech Inc. and Johns Hopkins University and EM], the University is entitled to milestone payments and royalties on sales of certain cancer vaccine products.

Reference information: J Clin Invest. 2018;128(8):3209-3218.

https://doi.org/10.1172/JCl120775. checkpoint molecules that can cause Teffs to become functionally "exhausted" in the context of chronic antigen exposure.

Cytotoxic T lymphocyte-associated protein 4 (CTLA-4) and its ligands B7.1 and B7.2 were the first checkpoints to be discovered (6). CTLA-4 acts early during $\mathrm{T}$ cell priming by competing with $\mathrm{CD} 28$ for the $\mathrm{B} 7$ receptor and thereby prevents $\mathrm{CD} 4^{+} \mathrm{T}$ cell activation. This discovery led to the realization that blocking CTLA-4 can override $\mathrm{T}$ cell desensitization to tumor antigens, hence, the development and approval of ipilimumab, a CTLA-4 antagonist antibody, for melanoma patients (7). Equally revolutionary has been the cloning and characterization of programmed cell death receptor 1 (PD-1) and its ligands, PD-L1 and PD-L2, on activated T cells $(8,9)$. Antibodies to PD-1, namely nivolumab and pembrolizumab, and PD-L1, such as atezolizumab, yield favorable clinical responses in melanoma, non-small cell lung cancer (NSCLC), mismatch repair-deficient (MMR-d) colorectal cancers, and renal cell carcinoma, among other cancers (10-14). These checkpoints are the first of many to be modulated to elicit antitumor immunity in patient tumors. A larger number of agents are already in various stages of clinical development.

\section{Barriers to checkpoint therapy}

Despite the established clinical efficacy of immune checkpoint inhibitors in a number of tumor types, several barriers prevent their overall utility. Important among these barriers is that the currently approved agents, when used as monotherapies, do not provide durable clinical responses in nearly $80 \%$ of cancer patients (15). Cancers that respond to checkpoint blockade usually already have significant numbers of $\mathrm{T}$ cells infiltrating their tumors, while cancers that do not naturally activate $\mathrm{T}$ cells for multiple reasons (including the lack of high mutational burdens within their tumors) 


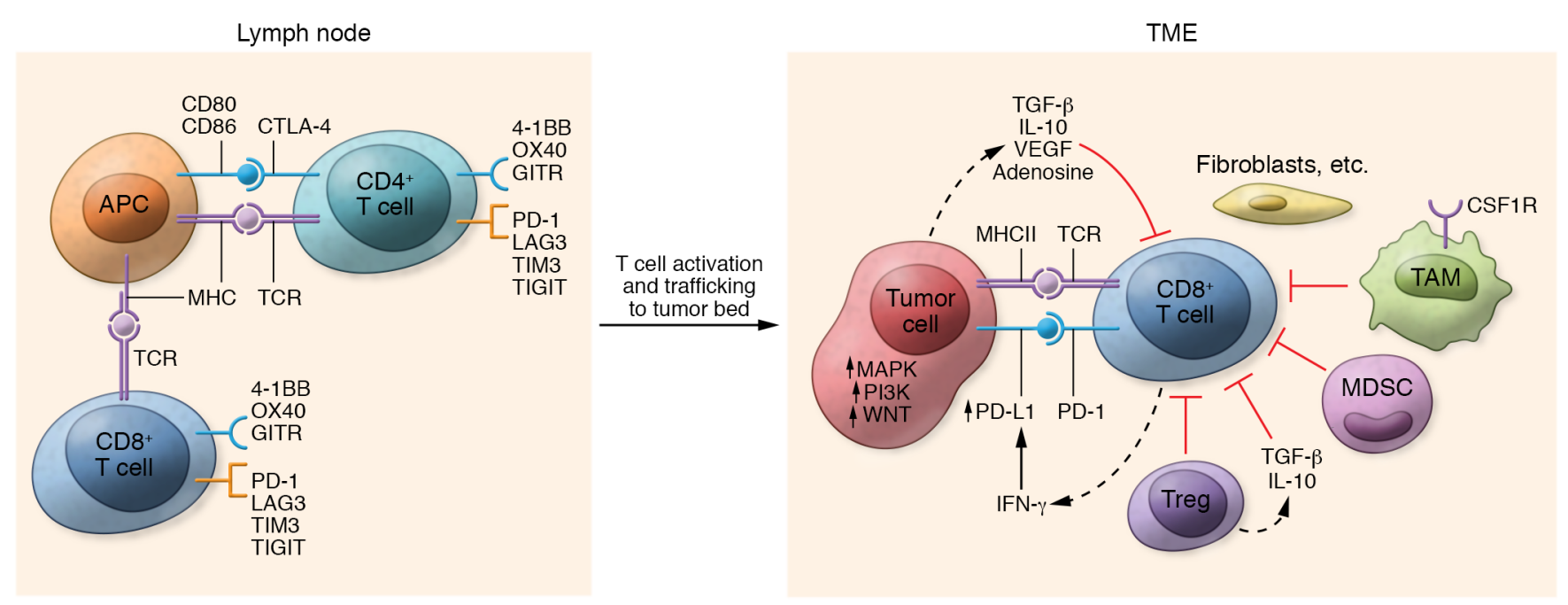

Figure 1. Resistance mechanisms to current checkpoint inhibitors. Factors that contribute to resistance to checkpoint inhibitors may be primary, adaptive, or acquired. In the lymph node, T cells are subject to precise regulation by both stimulatory (4-1BB, OX40, GITR) and inhibitory (PD-1, LAC3, TIM3, TIGIT, CTLA-4) checkpoints. The upregulation of inhibitory checkpoints may lead to T cell exhaustion. Furthermore, once T cells traffic to the TME, Teffs may be subject to multiple immunosuppressive signals by both immune cell subsets, such as M2-polarized tumor-associated macrophages (TAMs), Tregs, and MDSCs, and soluble mediators, including cytokines, such as TCF- $\beta$, IL-10, various chemokines, VEGF, adenosine, and ID01. Tumors may also recruit a stromal compartment consisting of fibroblasts and other cell types. Furthermore, as a mechanism of adaptive resistance, IFN may itself cause PD-L1 to become upregulated on tumor cells. Finally, genomic alterations within tumor cells may lead to resistance. These include increased oncogenic signaling through the MAPK pathway, loss of PTEN expression with enhanced PI3K signaling, and altered $\beta$-catenin, causing constitutive WNT signaling.

do not respond. There is, in fact, a positive correlation between a high burden of tumor neoantigens and response to immune checkpoint agents (16-18). Thus, one barrier to checkpoint therapy is the lack of available $\mathrm{T}$ cells that are capable of responding to immune checkpoint therapy (16-18).

A second barrier that needs to be understood is that initially responding cancers eventually become resistant to checkpoint agents through diverse genetic and immune-related mechanisms (4). For example, a loss of PTEN can activate PI3 kinase signaling (19), and JAK1/2 or STAT mutations downstream of IFN- $\gamma$ can impair $\mathrm{T}$ cell activation (20-22). Constitutive Wnt signaling through $\beta$-catenin activation can also lead to a paucity of $\mathrm{CD}_{103^{+}}$ dendritic cells and less inflamed tumors (4). Antigen presentation may also become reduced, for example, with $\beta_{2}$-microglobulin (B2M) mutations that lead to the loss of HLA molecules (22). Furthermore, the overall neoantigen landscape may evolve as immunogenic neoantigens are edited out of tumor cells (23).

A further layer of complexity resides in an altered tumor microenvironment (TME). The accrual of $\mathrm{CD}^{+} \mathrm{CD} 25^{+}$Tregs, myeloid-derived suppressor cells (MDSCs), and M2-polarized tumor-associated macrophages (TAMs) as well as the production of cytokines and immune metabolites may together render the TME immunosuppressive (Figure 1). This is particularly true for cancers that are naturally resistant to immune checkpoint agents. Tregs in the TME include "natural" Tregs, which differentiate in the thymus as a separate lineage that expresses Foxp3, as well as locally induced $\mathrm{T}$ regulatory 1 cells (TR1) that form when $\mathrm{CD} 4{ }^{+} \mathrm{CD} 25^{-}$cells are converted by exposure to high levels of TGF- $\beta$, IL-10, and other immunosuppressive cytokines (24). Tregs play a key immunosuppressive role in the TME as the main contributors to the induction and maintenance of $\mathrm{T}$ cell tolerance to tumor antigens (24). Additionally, Tregs express high levels of suppressive immune checkpoints, most notably CTLA-4 (24). MDSCs are another immunosuppressive cell population that is uniquely expanded during inflammation, infection, and cancer (25). Under these conditions, MDSCs upregulate their expression of immunosuppressive factors such as arginase 1 and inducible nitric oxide synthase (iNOS) as well as increase their production of nitric oxide (NO) and reactive oxygen species (ROS), which leads to suppression of T cells, macrophages, and dendritic cells (25). MDSCs contribute to Treg induction and upregulation of CTLA-4 expression on Tregs through the release of immunosuppressive cytokines, such as TGF- $\beta$ (25). The third main immunosuppressive cell population in the TME consists of TAMs, which exert numerous protumor effects, such as VEGF secretion to aid in angiogenesis; release of arginase I, IL-10, TGF- $\beta$, and other immunosuppressive factors; and the expression of PD-L1/2 and B7-1/2 (26). Finally, additional inhibitory checkpoint pathways, including metabolic enzymes such as idoleamine 2,3 dioxygenase (IDO), adenosine receptors, and inhibitory signals, such as CSF1R, become upregulated either initially or in response to checkpoint inhibitory therapy (Figure 1).

As only $20 \%$ of cancer patients respond to single-agent checkpoint inhibitors, there has been renewed interest in developing novel checkpoint modulators that can inactivate or activate $\mathrm{T}$ cell immunity to a therapeutic advantage (Figure 2). There is equally burgeoning interest in studying checkpoint combinations, such as nivolumab and ipilimumab, in advanced melanoma as a first example (27). Finally, studies have centered on combining current and newer checkpoints with other agents, such as cancer vaccines, that can first induce a "quality" $\mathrm{T}$ cell with the potential to respond to immune checkpoint agents. Here, we will discuss emerging checkpoint modulators and paradigms to combine checkpoints with other therapeutic strategies. 


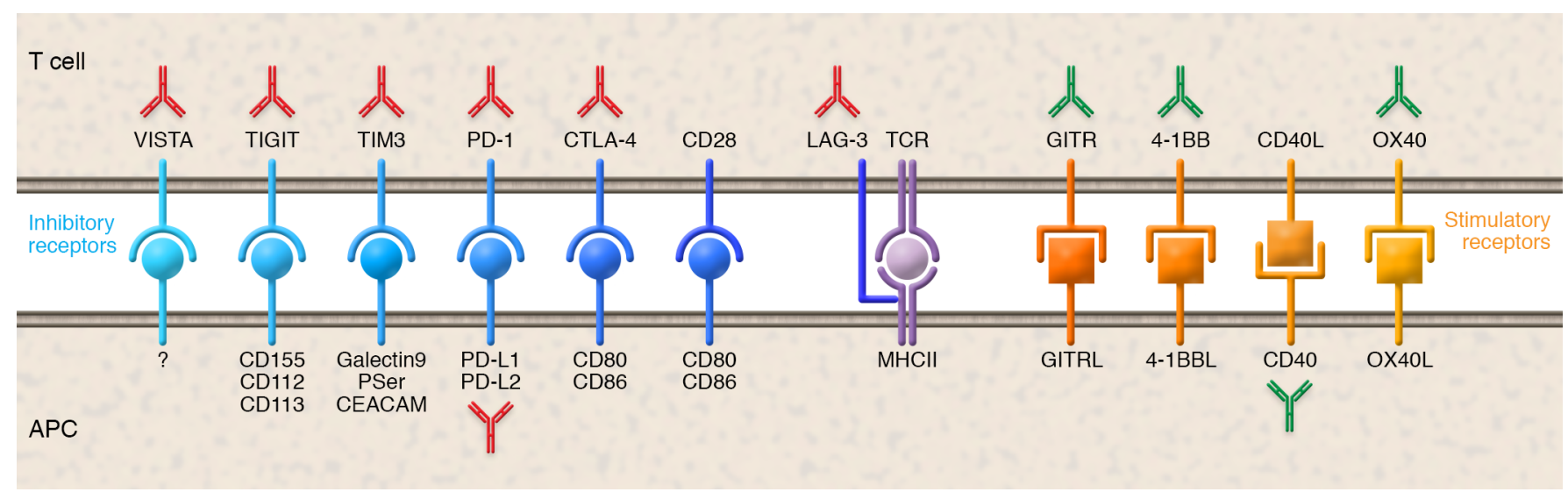

Figure 2. Checkpoints modulate T cell and antigen-presenting cell interactions. Either antagonist (red) or agonist (green) antibodies are currently under clinical testing based on promising preclinical data. Shown are the antibodies' interactions with ligand(s). Antibodies against PD-1 and CTLA-4 are FDA approved. The remaining antibodies are in clinical trials.

\section{Emerging immune checkpoints}

Inhibitory immune checkpoints. Several inhibitory checkpoints are currently being tested both as monotherapy and in combination with PD-1 blockade. Many of these inhibitors are upregulated on $\mathrm{T}$ cells to cause resistance, and their disruption may therefore enhance antitumor immunity. Here, we discuss four checkpoint inhibitor targets, namely lymphocyte-activation gene 3 (LAG-3), $\mathrm{T}$ cell immunoglobulin and mucin domain-containing molecule-3 (TIM-3), T cell immunoreceptor with immunoglobulin and ITIM domains (TIGIT), and V domain-containing Ig suppressor of $\mathrm{T}$ cell activation (VISTA).

LAG-3 is expressed on Teffs, Tregs, and dendritic cells, among other immune cells (28-30). It is homologous structurally to CD4 and therefore binds MHCII on APCs to transmit inhibitory signals that promote Treg-mediated immune suppression (31). LAG-3 is also expressed on cytotoxic $\mathrm{CD} 8^{+} \mathrm{T}$ cells to confer reduced proliferation and effector function. Its blockade by an anti-LAG-3 antibody or the genetic deletion of Lag3 in mice reverses these effects (32). Importantly, the coexpression of LAG-3 and PD-1 signals T cell exhaustion and tolerance to self and tumor antigens (33). By reversing these actions in synergy, anti-LAG-3 and anti-PD-1 antibodies together display enhanced clearance of tumors that were previously resistant to single checkpoint $(34,35)$. Consistent with pharmacologic blockade, compound Lag3 $3^{--}: \mathrm{Pd1}^{-/-}$mice also show efficient tumor clearance (35). Likewise, in ovarian cancer patients, patient-derived tumor-infiltrating NY-ESO-1-specific LAG- ${ }^{+} \mathrm{PD}-1^{+}$ $\mathrm{CD}^{+} \mathrm{T}$ cells are markedly dysfunctional, and coblockade of LAG-3 and PD-1 restores $\mathrm{T}$ cell proliferation and cytokine production (36). Preliminary results combining the anti-LAG-3 antibody BMS-986016 with nivolumab (ClinicalTrials.gov NCT01968109) showed an overall response rate (ORR) of $13 \%$ in melanoma patients who had relapsed on anti-PD-1 therapy (37). A soluble splice variant of LAG-3 that binds MHCII and displays immuneactivating properties has also been tested as an alternative LAG-3modulating agent (38), with favorable responses in a phase 1 renal cell carcinoma study (39). In summary, we believe that LAG-3 is most likely to work in synergy with anti-PD-1 to rescue T cells from exhaustion, although we await more mature clinical data.
TIM-3, an inhibitory immune receptor expressed on $\mathrm{CD}_{4}^{+}$and $\mathrm{CD} 8^{+} \mathrm{T}$ cells, Tregs, and dendritic cells, binds primarily to galectin-9 to trigger $\mathrm{T}$ cell apoptosis $(40,41)$. It also binds an alternative ligand, CEACAM-1, the expression of which is upregulated by IFN- $\gamma$ (ref. 42 and Figure 2). The interaction between TIM-3 and galectin-9 has been shown to negatively regulate $\mathrm{T}$ helper type 1 responses, which contribute to the induction of peripheral tolerance $(41,43)$. Furthermore, the expression of TIM-3 on innate immune cells, such as dendritic cells and monocytes, plays a role in the promotion of tissue inflammation (44). In mice transplanted with colon cancer (CT26) cells, tumor-infiltrating TIM- $3^{+} \mathrm{PD}-1^{+} \mathrm{T}$ cells were found to display the most exhausted phenotype $(45,46)$. In these mice, as well as in a carcinogen-induced sarcoma model, anti-TIM-3 and anti-PD-1 antibodies injected together resulted in marked tumor regression and restored cytokine production (46, 47). Similarly, in melanoma patients, $\mathrm{TIM}-3^{+} \mathrm{CD} 4^{+}$and TIM- $3^{+}$ $\mathrm{CD}^{+} \mathrm{T}$ cells were found to contribute to the immunosuppressive TME $(48,49)$, and an anti-TIM-3 antibody restored T cell function (48). Further evidence points to an upregulated TIM-3 pathway in the setting of acquired resistance to PD-1 blockade, as noted both in Egfr ${ }^{\mathrm{T} 790 \mathrm{M} / \mathrm{L} 858 \mathrm{R}}: \mathrm{Kras}^{\mathrm{G} 12 \mathrm{D}}$ mice (a model for lung adenocarcinoma) and in two patients with NSCLC (50). An antagonist TIM-3 antibody given upon relapse improved survival in mice (50). At least three phase I trials are currently evaluating the efficacy of combining anti-TIM-3 with anti-PD-1 or anti-PD-L1 therapy in advanced solid malignancies (NCT02817633, NCT03099109, and NCT02608268). Similarly to Lag-3, an antibody against TIM-3 would likely work to reinvigorate $\mathrm{T}$ cells, leading to a less exhausted phenotype, and therefore promote antitumor immunity.

A member of the family of poliovirus receptors, TIGIT is expressed on effector $\mathrm{CD} 4^{+}$and $\mathrm{CD} 8^{+} \mathrm{T}$ cells, Tregs, and NK cells (51). It exerts potent immune inhibition through high-affinity binding to CD155 and interacts with lower affinities with CD112 and other cognate ligands (Figure 2). Similar to the CTLA-4 and CD28 competition, TIGIT competes with nectin family proteins, namely CD155 and CD112, for the immune-activating receptor CD226 and, in doing so, blocks this otherwise stimulatory pathway $(51,52)$. The suppression of antitumor immunity occurs not 
only through highly immunosuppressive TIGIT $^{+}$Tregs, but also via the direct killing of $\mathrm{CD}^{+} \mathrm{T}$ cells and NK cells (53). Furthermore, tumor-infiltrating $\mathrm{TIGIT}^{\mathrm{hi}} \mathrm{CD}^{+} \mathrm{T}$ cells display an exhausted $\mathrm{T}$ cell phenotype and coexpress other inhibitory checkpoints, namely PD-1, TIM-3, and LAG-3 $(53,54)$. Coblockade of TIGIT and either PD-1 or TIM-3 enhances antitumor activity in the CT26 mouse model $(54,55)$. TIGIT targeting is still early in clinical development, with an antagonist anti-TIGIT antibody, OMP313M32 (NCT03119428), being evaluated as a single agent and two antibodies, MTIG7192A (NCT02794571) and BMS-986207 (NCT02913313), being evaluated in combination with PD-1 blockade. Overall, anti-TIGIT is a newer antibody that has the unique potential to activate cytotoxic T cells and NK cells and is therefore likely to elicit tumor cell killing through multiple pathways.

A newer checkpoint, VISTA, is structurally homologous to PD-L1 and is found primarily on myeloid APCs and T cells, in particular on Tregs $(56,57)$. VISTA enhances Treg maturation and inhibits $\mathrm{T}$ cell activation and hence contributes to an immunosuppressive TME $(56,57)$. Its blockade expectedly decreases Tregs (and MDSCs) in the TME, activates dendritic cells, causes tumor regression, and improves survival in mouse models (58-60). VISTA, PD-1, and PD-L1 have also been shown to be coupregulated in tumor-infiltrating T cells and M2 macrophages from localized and metastatic prostate cancer patients treated with ipilimumab (61). To date, there is only one ongoing clinical trial utilizing an anti-VISTA monoclonal antibody, JNJ-61610588 (NCT02671955). It is early in our understanding of VISTA, but the checkpoint shows promise in reprogramming several different components of the TME, including MDSCs, Tregs, and M2-macrophages.

Stimulatory immune checkpoints. Stimulatory checkpoints targeted by agonist antibodies against the respective molecules are likely to be most beneficial in amplifying preexisting $\mathrm{T}$ cell responses unleashed by PD-1/PD-L1 disruption. The use of agonist antibodies results in enhanced immunologic memory and a more robust clinical response. Several stimulatory checkpoints, namely OX40, glucocorticoid-induced TNF receptor-related protein (GITR), T cell antigen 4-1BB homologue (4-1BB), CD40, and inducible $\mathrm{T}$ cell costimulator (ICOS), are being tested in preclinical and clinical settings.

OX40, also known as TNFRSF4 or CD134, is a TNF receptor (TNFR) superfamily member that is expressed on all T cell subsets, particularly on Tregs and NK cells, whereas its ligand OX4OL is found on APCs (38). OX40 expression is transient - it is upregulated approximately 12 hours after $\mathrm{T}$ cell activation and declines by day 4. The primary functions of OX40 are to promote T cell survival, proliferation, and memory, enhance cytokine secretion, and deplete Tregs in the tumor (62-65). Of utmost importance is that OX40 is critical to CD4 ${ }^{+} \mathrm{T}$ cell responses and long-term memory. Not only does OX40 regulate the number of $\mathrm{CD} 4^{+} \mathrm{T}$ cells that can be generated during a primary clonal expansion, but it also promotes the number of $\mathrm{CD} 4^{+} \mathrm{T}$ cells that can persist as memory cells (66). This is pivotal, as it is now becoming clear that the quality of $\mathrm{T}$ cells is as important as $\mathrm{T}$ cell number in determining response to immunotherapy (67). OX40 has also been shown to improve the survival of low-avidity, tumor-reactive T cells, thus further enhancing antitumor immunity (62). Finally, the interaction between OX40 on T cells and OX4OL on dendritic cells plays a critical role in dendritic cell activation and maturation $(68,69)$. Multiple preclinical models have documented efficacy of an agonist anti-OX40 antibody both as monotherapy (70) and in combination with other immunomodulatory antibodies to 4-1BB, PD-1, and TIM-3 (71-73). The first human trial using a mouse OX40 agonist did not meet objective RECIST criteria (established benchmarks for evaluating responses in solid tumors), likely due to the induction of antimouse immunity (74). Since then, multiple humanized antibodies have been developed and are currently undergoing clinical testing. Preliminary data from one such antibody, MOXR0916, in combination with atezolizumab have demonstrated safety in a phase $1 \mathrm{~b}$ trial (NCT02410512) (75). We surmise that by activating memory and better "quality" T cells, OX40 is most likely to be a potent combinatorial partner to checkpoint inhibition.

An OX40-like molecule, GITR (also known as TNFRS18 or CD257), also belonging to the TNFR family, displays a delayed upregulation at around 24 to 72 hours after T cell activation. GITR stimulation leads to enhanced Teff proliferation and cytokine production (76). Similarly to OX40, its delayed expression suggests that GITR does not play a significant role in T cell priming, but instead acts shortly thereafter. GITR is also constitutively expressed on Tregs at high levels and impairs Treg ingress into tumors $(77,78)$. An agonist GITR antibody, DTA-1, has demonstrated efficacy as a monotherapy in a melanoma mouse model (79) as well as in combination with PD-1 or CTLA-4 blockade in models of colon cancer and fibrosarcoma $(80,81)$. While we await more mature clinical data from early phase trials, preliminary results using an agonist anti-GITR antibody, TRX-518, in a dose-escalation study show safety in advanced malignancies (NCT01239134). Thus, GITR agonism will likely have a role in tumors in which Tregs are major immunosuppressive components in the TME, given its dual role in impairing Treg function and ingress into tumors while also enhancing Teff activity.

A third TNFR family checkpoint, 4-1BB (also known as CD137 or TNFRS9), is expressed transiently and early after $\mathrm{T}$ cell activation (82). While 4-1BB is expressed widely on activated T cell subsets, NK cells, Tregs, and innate immune cells, including monocytes, its ligand, $4-1 \mathrm{BBL}$, is localized to dendritic cells. 4-1BB regulates antiapoptotic genes, including $B c l-x l$ and $B f l-1$, via $\mathrm{NF}-\kappa \mathrm{B}$ and, in doing so, increases $\mathrm{T}$ cell survival $(83,84)$. Blockade of 4-1BB with antagonist antibodies also synergizes with other checkpoint blockades; for example, 4-1BB- and PD-1- targeting combination therapy caused tumor regression in an ovarian cancer model $(85,86)$. Agonist anti-4-1BB antibodies, namely urelemab (IgG4) and PF-582566 (IgG2), are currently being tested in early phase clinical studies. A phase II study of urelemab given at $1 \mathrm{mg} /$ $\mathrm{kg}$ to metastatic melanoma patients was terminated early due to grade 4 hepatotoxicity, but further safety analysis confirmed safety at a lower dose of $0.1 \mathrm{mg} / \mathrm{kg}$ every three weeks (87). In summary, $4-1 \mathrm{BB}$ still requires careful evaluation of toxicity, particularly as combinatorial therapy.

CD40, also a TNFR family member, is expressed on B cells, macrophages, dendritic cells, and certain tumor cells, whereas its ligand, CD4OL (also known as CD154), is present on activated $\mathrm{CD}^{+} \mathrm{T}$ cells (88). CD40 activation upregulates MHCII on APCs and elicits the secretion of proinflammatory cytokines, including IL-12, and thereby primes tumor-specific $\mathrm{CD}^{+} \mathrm{T}$ cell responses 
A

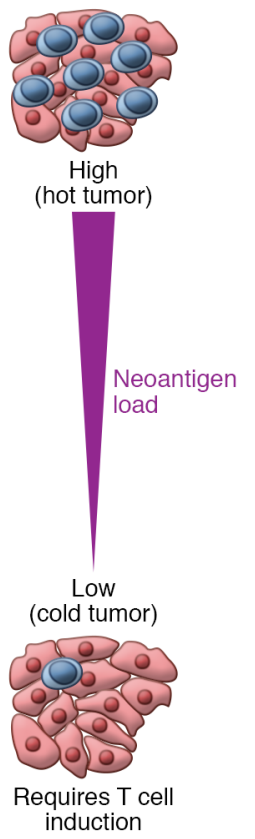

B

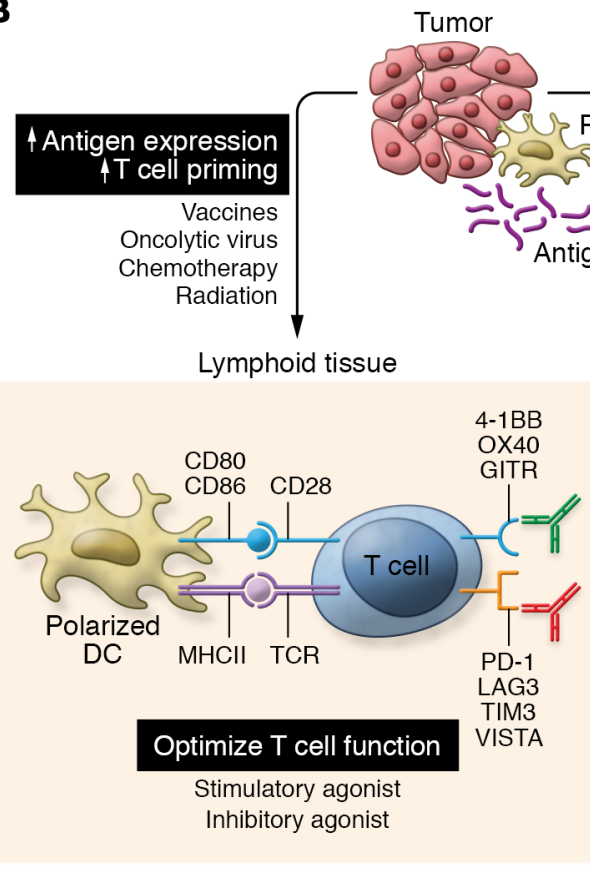

$\downarrow_{\mathrm{TME}}$

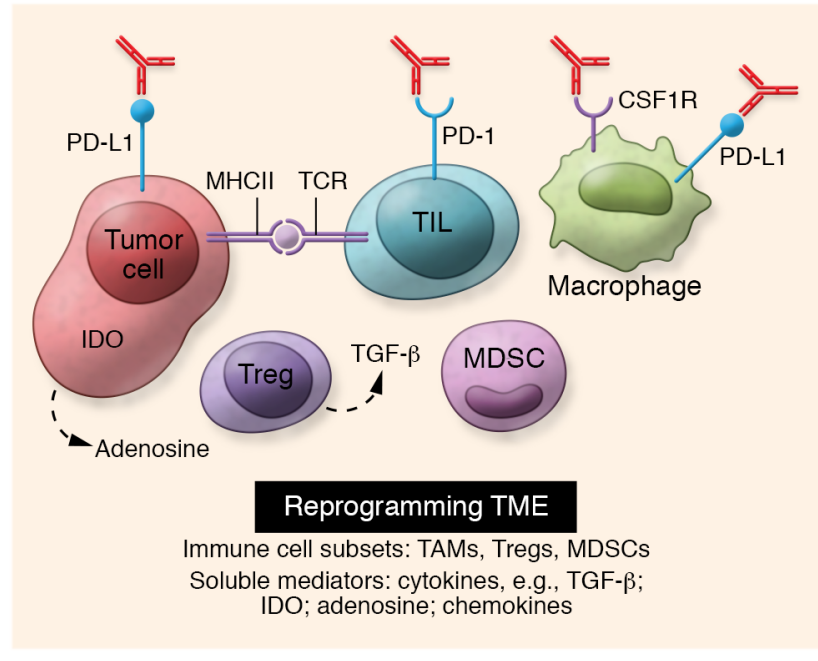

Figure 3. Combinatorial therapy of checkpoint modulators with other anticancer modalities. (A) Immunologically hot and cold tumors differ in neoantigen burden. Hot tumors just need checkpoint inhibitors or combinations of checkpoint inhibitors and/or checkpoint agonist antibodies to optimize T cell function. However, in addition to checkpoint modulation, naturally cold tumors require T cells to be first primed and then to traffic to the tumor tissue. (B) Several strategies exist to induce priming of T cells and/or enhance antigen expression, such as vaccines, oncolytic viruses, chemotherapy, and radiation. Polarized dendritic cells then traffic to the lymph node and activate T cells, a process that is regulated precisely by stimulatory (e.g., OX40, GITR, 4-1BB) and inhibitory checkpoints (e.g., PD-1, LAG-3, TIM3, VISTA), which can enhance or inhibit T cell responses, respectively. These checkpoints can be modulated by their respective antibodies that are currently being tested clinically. Many cold tumors also require reprogramming of other immune subsets in the TME. Tumors recruit immunosuppressive cells, such as Tregs, MDSCs, and M2-polarized macrophages, which can be modified via various strategies. The action of soluble mediators, such as adenosine, IDO, cytokines (TCF- $\beta$ ), and chemokines, can also be modulated.

(89-92). CD40 ligation on B cells also causes increased antigen presentation and proliferation (92). Impressive results have been noted with CD40 agonism in pancreatic cancers, where low $\mathrm{T}$ cell numbers, high numbers of TAMs, and an overall immunosuppressive stroma are hallmarks of immune resistance. In a $\mathrm{Kras}^{\mathrm{LSL}-\mathrm{G} 12 \mathrm{D} /+}$ : Trp53 $3^{\mathrm{LSL}-\mathrm{R} 172 \mathrm{H} /+}:$ Pdx1-Cre (KPC) transgenic mouse model of pancreatic adenocarcinoma, an agonist CD40 antibody with gemcitabine caused the depletion of tumor stroma and triggered the rapid infiltration of $\mathrm{CD} 4 \mathrm{O}^{+}$-activated macrophages that were reprogrammed to a tumoricidal M2 phenotype (93). In the same KPC mouse model, a single dose of agonist CD40 antibody in conjunction with standard chemotherapy, namely nab-paclitaxel and gemcitabine, converted a $\mathrm{T}$ cell-poor to a $\mathrm{T}$ cell-infiltrated tumor, resulting in durable control, whereas neither treatment modality alone elicited a $\mathrm{T}$ cell response (94). Among several other trials, anti-CD40 is currently being tested with gemcitabine and nab-paclitaxel in pancreatic cancer patients with resectable disease (NCT02588443). CD40 agonist combinatorial therapy is thus likely to have a major role in converting immunologically "cold" into "hot" tumors.

\section{Combinatorial strategies}

Effective antitumor immunity results from antigen-specific $\mathrm{T}$ cell priming in lymphoid tissue, Teff differentiation, trafficking of $\mathrm{T}$ cells to the tumor bed, and finally, killing of tumor cells by cytotox- ic $\mathrm{CD} 8^{+} \mathrm{T}$ cells. Immunosuppressive signals in the TME can cause tumor-infiltrating lymphocytes (TILs) to be exhausted or dysfunctional, leading to tumor escape and therapeutic resistance. In many instances, monotherapy with checkpoint modulators may not sufficiently intercept these inhibitory signals. Thus, combinations of checkpoint antibodies may be required to enhance tumor immunogenicity. In other instances, such as in pancreatic cancers, which do not naturally attract $\mathrm{T}$ cells, checkpoints may need to be paired with other agents that would first induce tumor-specific TILs.

Inducing $T$ cells into an immunologically cold tumor. The conversion of an immunologically cold or noninflamed tumor to a hot tumor merits consideration of three broad, but overlapping, approaches (Figure 3). First, $T$ cells need to be primed sufficiently with antigen in the lymph node and then must traffic to and infiltrate the tumor. One strategy to induce $\mathrm{T}$ cell clones or broaden the $\mathrm{T}$ cell repertoire uses vaccines, such as the granulocytemacrophage CSF-secreting (GM-CSF-secreting) allogenic vaccine GVAX, particularly in tumors that are neoantigen-low, such as pancreatic cancer. We showed that, when given alone, GVAX increased lymphoid aggregates in the pancreatic tumors, while, in combination with ipilimumab, it enhanced antitumor immunity (95). New approaches using personalized, neoantigen-targeted vaccines are being explored as attractive modalities for inducing neoepitope-specific $\mathrm{T}$ cells that are less likely to be deleted by central and peripheral tolerance due to their lack of expression in 
the thymus and their relatively recent expression in the periphery, respectively $(96,97)$. Our group and others have also shown that, while inducing tumor-specific $\mathrm{T}$ cells, cancer vaccines, when administered alone, can upregulate immunosuppressive pathways, such as PD-L1, mainly through the increased secretion of IFN- $\gamma$ (98). This means that cancer vaccines are likely to work best when in combination with checkpoint modulation.

A second approach is to use oncolytic viruses that enhance $\mathrm{T}$ cell priming, which have shown efficacy, particularly in melanoma patients $(99,100)$. Briefly, these virus strains preferentially attack tumor cells, causing the release of tumor antigens and thereby enhancing antigen expression. Talimogene laherparepvec (T-VEC), a herpes simplex 1-derived virus, replicates within tumors and produces GM-CSF to enhance antitumor immunity. A significant advantage in overall survival (OS) was noted with T-VEC versus GM-CSF in a randomized open-label phase III trial of patients with metastatic, unresectable melanoma (99).

A third combinatorial strategy uses checkpoints with chemotherapy and radiation therapy. Radiation therapy increases the expression and/or release of tumor neoantigens, which results in greater antigen presentation and enhanced antitumor immunity (101). There is evidence for synergy and an enhanced T cell repertoire in tumors when radiation therapy is combined with CTLA-4 or PD-1 blockade in patients with metastatic melanoma (102, 103). Chemotherapy likewise causes cell death and releases tumor antigens to enhance antigen presentation. The widely used chemotherapeutics pemetrexed and carboplatin have recently been approved for use in combination with pembrolizumab for the firstline treatment of NSCLCs, notably irrespective of PD-L1 expression. There was an impressive increase in ORR of $55 \%$ in the combinatorial group (pemetrexed, carboplatin, and pembrolizumab) versus $29 \%$ with chemotherapy alone (104). Another mechanism for chemotherapy-induced enhancement of tumor immunity is the alteration of immune subsets in the TME, as discussed below. For example, cyclophosphamide has been used at a low dose to deplete Tregs, and paclitaxel, fluorouracil (5FU), and taxanes to decrease MDSCs $(105,106)$.

Optimizing $T$ cell function. T cells primed with tumor antigen and subjected to chronic antigen exposure may eventually need rescue from exhaustion with checkpoint-modulating agents (Figure 3). Combining ipilimumab and nivolumab yields a significant OS benefit when compared with ipilimumab alone in patients with metastatic melanoma (27). Stimulatory checkpoints, such as OX40, GITR, and CD40, may have a role in further enhancing Teff function. As noted above, as most costimulatory checkpoints, notably OX40 and GITR, are expressed after initial T cell priming, they may not be effective as monotherapy. Instead, their modulation may be more helpful in amplifying a preexisting $\mathrm{T}$ cell response and in generating a memory response. Similarly, checkpoint inhibitors, importantly, LAG-3 and TIM-3, may only be useful in tumors that have preexisting tumor-specific $\mathrm{T}$ cells that require reversal of exhaustion and restoration of $\mathrm{T}$ cell function. Thus, checkpoint modulators, even when used in combination, may not be sufficient to convert nonimmunogenic tumors into immunogenically hot tumors.

Reprogramming the TME. Residing within tumors is a highly complex microenvironment characterized by the presence of multiple immunosuppressive cell subsets, including Tregs, TAMs, and MDSCs; immunomodulatory cytokines, such as TGF- $\beta$; chemokines and their receptors, namely CXCL12 and CXCR4; and metabolic enzymes, such as IDO. In some tumor types, this highly immunosuppressed TME needs to be sufficiently reprogrammed to allow for a robust immune response (Figure 3). Toward this goal, several specific strategies are being explored in combination with checkpoint-modulating agents. Of note is that checkpoint modulators can themselves regulate the proliferation, function, and/or survival of Tregs, NK cells, and monocytes, among other immune cells.

There is significant new interest in using specific macrophage modulators to optimize TAMs, which otherwise mediate immune escape and treatment resistance. M2-polarized TAMs (as opposed to M1-polarized TAMs) promote tumorigenesis by inhibiting $\mathrm{T}$ cell function and secreting immunosuppressive cytokines and chemokines. CSF-1 is a macrophage-derived cytokine that acts upon its receptor, CSF1R, to maintain M2 polarization and induce TAM proliferation. An anti-CSF1R antibody has been shown to reprogram TAM polarization and to act in synergy with checkpoint blockade and gemcitabine in pancreatic cancer (107). Macrophage ingress into tumors is facilitated by two chemokines, CXCL12 and CCL2, which interact, respectively, with their receptors CXCR4 and CCR2. Anti-CXCR4 and anti-CCR2 antibodies inhibit TAM recruitment into tumors, but a phase 2 open-label trial did not provide evidence for an OS advantage by adding anti-CXCR4 antibody to sunitinib as first-line treatment for metastatic renal cell carcinoma (108).

MDSCs promote tumor cell invasion and metastases $(109,110)$ by suppressing antigen-specific $\mathrm{T}$ cell proliferation and inducing Tregs in the TME (25). Interestingly, greater MDSC numbers in patients with melanoma correlate with a poor ipilimumab response, indicating that MDSCs dampen the effect of checkpoint blockade (111). In this regard, strategies are focused on impairing MDSC function and depleting and/or reprograming MDSCs to enhance the efficacy of checkpoint agents. Etinostat, a histone deacetylase (HDAC) inhibitor, is one example of an agent that impairs MDSC function, enhances antitumor immunity, reduces tumor growth, and increases survival when used in combination with anti-PD-1 antibody in lung and renal cell carcinoma models (112). In the ongoing phase II ENCORE 601 trial, preliminary results with the PD-1/HDAC inhibitor combination induced a favorable response in 4 of 13 (31\%) melanoma patients who had progressed on checkpoint inhibitor monotherapy (NCT02437136) (113).

Finally, a host of other molecules released locally by immune and tumor cells have been found to contribute to immunosuppression and have therefore been targeted toward TME reprogramming. TGF- $\beta$, produced by TAMs and tumor cells, is one cytokine that contributes to Treg activation and tumor angiogenesis. Increased levels of TGF- $\beta$ are associated with poor prognosis, and TGF- $\beta$ inhibitors have been shown to synergize with anti-CTLA-4 antibodies in melanoma models to enhance antitumor immunity (114). We likewise found that combining GVAX and TGF- $\beta$ blockade resulted in the depletion of Tregs and a survival advantage in a Treg-rich pancreatic cancer model (115). Another immunosuppressive molecule, IDO1, is a tryptophan-metabolizing enzyme produced by tumor cells, TAMs, and MDSCs. IDO1 negatively 
affects Teff function and enhances Treg activity $(116,117)$. Similarly to the IFN- $\gamma$-induced upregulation of PD-L1 and CEACAM-1, IFN- $\gamma$ also induces IDO1, thereby contributing to resistance to checkpoint blockade (118). Several IDO inhibitors are thus being evaluated clinically. The most advanced evaluation is in the phase III trial combining the IDO inhibitor epacadostat with pembrolizumab in patients with unresectable or metastatic melanoma (NCT02752074), results of which are awaited.

Testing combinatorial immunotherapies. Novel combinatorial therapies with immune checkpoint modulators are required to both enhance response rates in hot tumors and to sensitize cold tumors to immunotherapy. However, there is an unmet need for the rational design of clinical trials that are informed by rigorous preclinical testing and a deep mechanistic understanding of both monotherapies and combinatorial approaches. For example, certain agonist molecules, such as an agonist OX40 antibody, may only work early, just following $\mathrm{T}$ cell activation, and thus sequencing of immune-modulating agents may be pivotal in such combinatorial trials. Optimal clinical trial design would also require a full understanding of drug pharmacokinetics in order to reduce the possibility of toxicity. Furthermore, in early phase clinical trials, there is a requirement for multiple biopsy time points so that we can understand the changes in the immune milieu over time with treatment. Finally, overlapping toxicities of immunotherapies must be considered carefully when combining modalities.

A second requirement for optimal combinatorial immunotherapy is the identification of sensitive biomarkers for guidance toward individuals who would benefit most from immune checkpoint modulators and minimize unnecessary toxicities in those unlikely to respond. Novel response biomarkers to combination therapy are emerging, although most, to date, have studied the effect of anti-PD-1 monotherapy. Most notable among these are tumor PD-L1 expression and tumor-mutational burden (TMB), which have both been shown to predict anti-PD-1 responses $(16-18,119)$. Most recently, TMB has been shown to be a strong predictor of objective response, durable benefit, and progression-free survival (PFS) in patients with NSCLC who had received combined anti-PD-1 and anti-CTLA-4 blockade (119). MMR deficiencies, which lead to greater TMB and neoantigen load, have likewise been extraordinary predictors of anti-PD-1 inhibition (11, 12). This predictive biomarker was initially described as predicting response in a subset of colon cancer, but has now been applied across all tumor types $(11,12)$.

Finally, there are new data to suggest that higher quality preexisting T cells may predict response to chimeric antigen receptor (CAR) T cell therapy in patients with chronic lymphocytic leukemia (CLL) (67). Those patients enriched in early memory CD8 ${ }^{+} \mathrm{T}$ cells $\left(\mathrm{CD} 27^{+} \mathrm{CD} 45 \mathrm{RO}^{-}\right)$showed a complete or partial response to CAR T cell therapy, whereas patients without this subset did not respond
(67). Transcriptome analyses of responders versus nonresponders demonstrated that the CAR $\mathrm{T}$ cells that persisted in responders were enriched in genes that regulate early memory and Teffs and possess the $I L-6 / S T A T 3$ gene signature, while nonresponders expressed genes involved in late $\mathrm{T}$ cell differentiation, glycolysis, exhaustion, and apoptosis (67). Further studies that evaluate the quality of $\mathrm{T}$ cells before and after immunotherapies are thus warranted, as it is becoming increasingly clear that it is not just the number of $\mathrm{T}$ cells infiltrating the tumor, but also their quality, that influences the overall antitumor immune response. In summary, the horizon of biomarker development is diversifying and broadening, and it is likely that multiple biomarkers will cumulatively predict response to mono- and combination immunotherapy.

\section{Conclusions}

Immune checkpoint-modulating agents have proven to be increasingly successful in driving antitumor immune responses, particularly for those tumors, such as melanoma, that harbor high neoantigen loads and thus naturally attract $\mathrm{T}$ cells into the tumor. In such tumor types, combinations of checkpoint-modulating agents have been successful in increasing antitumor immunity. However, T cell exhaustion due to multiple upregulated inhibitory checkpoints limits their durable control of tumors. Additionally, there are many tumors, particularly those with a low neoantigen burden, that display primary resistance to checkpoint agents. An immunosuppressive TME further impairs antitumor immunity. It is therefore critical to convert immunologically cold tumors to $\mathrm{T}$ cell-rich hot tumors. Agents or strategies that activate $\mathrm{T}$ cells, reverse $\mathrm{T}$ cell exhaustion, and/or reprogram an otherwise immunosuppressive TME must be employed together with checkpoint modulators to achieve a robust and durable clinical response. Such combinatorial therapies are being tested widely, with a number of promising candidates due for FDA approval.

\section{Acknowledgments}

The authors gratefully acknowledge the Skip Viragh Center for Pancreas Cancer at Johns Hopkins and the Bloomberg-Kimmel Institute for Cancer Immunotherapy at Johns Hopkins. EMJ acknowledges support from the NIH (R01 CA184926) and Stand Up To Cancer-Lustgarten Foundation Pancreatic Cancer Convergence Team (SU2C-AACR-DT1414). NZ is supported in part by NIH grant T32 CA00971-371 and the 2018 American Society Clinical Oncology Young Investigator Award.

Address correspondence to: Neeha Zaidi, The Sidney Kimmel Comprehensive Cancer Center at Johns Hopkins, 4M Bunting Blaustein Cancer Research Building, 1650 Orleans Street, Baltimore, Maryland 21231, USA. Phone: 410.955.2957; Email: nzaidi1@jhmi.edu.
1. Coley WB. The treatment of inoperable sarcoma by bacterial toxins (the mixed toxins of the Streptococcus erysipelas and the Bacillus prodigiosus). Proc R Soc Med. 1910;3(Surg Sect):1-48.

2. Burnet FM. Immunological surveillance in neoplasia. Transplant Rev. 1971;7:3-25.

3. Dunn GP, Bruce AT, Ikeda H, Old LJ, Schreiber
RD. Cancer immunoediting: from immunosurveillance to tumor escape. Nat Immunol. 2002;3(11):991-998.

4. Sharma P, Hu-Lieskovan S, Wargo JA, Ribas A. Primary, adaptive, and acquired resistance to cancer immunotherapy. Cell. 2017;168(4):707-723.

5. Yarchoan M, Johnson BA 3rd, Lutz ER, Laheru
DA, Jaffee EM. Targeting neoantigens to augment antitumour immunity. Nat Rev Cancer. 2017;17(4):209-222.

6. Brunet JF, et al. A new member of the immunoglobulin superfamily - CTLA-4. Nature. 1987;328(6127):267-270.

7. Hodi FS, et al. Improved survival with ipilimumab 
in patients with metastatic melanoma. $N$ Engl J Med. 2010;363(8):711-723.

8. Freeman GJ, et al. Engagement of the PD-1 immunoinhibitory receptor by a novel B7 family member leads to negative regulation of lymphocyte activation. JExp Med. 2000;192(7):1027-1034.

9. Ishida Y, Agata Y, Shibahara K, Honjo T. Induced expression of PD-1, a novel member of the immunoglobulin gene superfamily, upon programmed cell death. EMBO J. 1992;11(11):3887-3895.

10. Garon EB, et al. Pembrolizumab for the treatment of non-small-cell lung cancer. $N$ Engl J Med. 2015;372(21):2018-2028.

11. Le DT, et al. Mismatch repair deficiency predicts response of solid tumors to PD-1 blockade. Science. 2017;357(6349):409-413.

12. Le DT, et al. PD-1 blockade in tumors with mismatch-repair deficiency. N Engl J Med. 2015;372(26):2509-2520.

13. Motzer RJ, et al. Nivolumab versus Everolimus in advanced renal-cell carcinoma. $N$ Engl J Med. 2015;373(19):1803-1813.

14. Robert C, et al. Pembrolizumab versus Ipilimumab in advanced melanoma. $N$ Engl J Med. 2015;372(26):2521-2532.

15. Ott PA, Hodi FS, Kaufman HL, Wigginton JM, Wolchok JD. Combination immunotherapy: a road map. J Immunother Cancer. 2017;5:16.

16. Rizvi NA, et al. Cancer immunology. Mutational landscape determines sensitivity to PD-1 blockade in non-small cell lung cancer. Science. 2015;348(6230):124-128.

17. Snyder A, et al. Genetic basis for clinical response to CTLA-4 blockade in melanoma. N Engl J Med. 2014;371(23):2189-2199.

18. Yarchoan M, Hopkins A, Jaffee EM. Tumor mutational burden and response rate to PD-1 inhibition. N Engl J Med. 2017;377(25):2500-2501.

19. Peng W, et al. Loss of PTEN promotes resistance to T cell-mediated immunotherapy. Cancer Discov. 2016;6(2):202-216.

20. Gao J, et al. Loss of IFN- $\gamma$ pathway genes in tumor cells as a mechanism of resistance to antiCTLA-4 therapy. Cell. 2016;167(2):397-404.e9.

21. Green MR, et al. Integrative analysis reveals selective 9p24.1 amplification, increased PD-1 ligand expression, and further induction via JAK2 in nodular sclerosing Hodgkin lymphoma and primary mediastinal large B-cell lymphoma. Blood. 2010;116(17):3268-3277.

22. Zaretsky JM, et al. Mutations associated with acquired resistance to PD-1 blockade in melanoma. N Engl J Med. 2016;375(9):819-829.

23. Anagnostou V, et al. Evolution of neoantigen landscape during immune checkpoint blockade in non-small cell lung cancer. Cancer Discov. 2017;7(3):264-276.

24. Zou W. Regulatory T cells, tumour immunity and immunotherapy. Nat Rev Immunol. 2006;6(4):295-307.

25. Gabrilovich DI, Nagaraj S. Myeloid-derived suppressor cells as regulators of the immune system. Nat Rev Immunol. 2009;9(3):162-174.

26. Noy R, Pollard JW. Tumor-associated macrophages: from mechanisms to therapy. Immunity. 2014;41(1):49-61.

27. Wolchok JD, et al. Overall survival with combined nivolumab and ipilimumab in advanced melano- ma. N Engl J Med. 2017;377(14):1345-1356.

28. Goldberg MV, Drake CG. LAG-3 in cancer immunotherapy. Curr Top Microbiol Immunol. 2011;344:269-278.

29. Huard B, et al. Characterization of the major histocompatibility complex class II binding site on LAG-3 protein. Proc Natl Acad Sci U S A. 1997;94(11):5744-5749.

30. Triebel F, et al. LAG-3, a novel lymphocyte activation gene closely related to CD4.JExp Med. 1990;171(5):1393-1405.

31. Huard B, Prigent P, Tournier M, Bruniquel D, Triebel F. CD4/major histocompatibility complex class II interaction analyzed with $\mathrm{CD} 4^{-}$and lymphocyte activation gene-3 (LAG-3)-Ig fusion proteins. Eur J Immunol. 1995;25(9):2718-2721.

32. Grosso JF, et al. LAG-3 regulates $\mathrm{CD}^{+} \mathrm{T}$ cell accumulation and effector function in murine self- and tumor-tolerance systems. JClin Invest. 2007;117(11):3383-3392.

33. Grosso JF, et al. Functionally distinct LAG-3 and PD- 1 subsets on activated and chronically stimulated CD8 T cells. JImmunol. 2009;182(11):6659-6669.

34. Huang RY, Eppolito C, Lele S, Shrikant P, Matsuzaki J, Odunsi K. LAG3 and PD1 co-inhibitory molecules collaborate to limit $\mathrm{CD} 8^{+} \mathrm{T}$ cell signaling and dampen antitumor immunity in a murine ovarian cancer model. Oncotarget. 2015;6(29):27359-27377.

35. Woo SR, et al. Immune inhibitory molecules LAG-3 and PD-1 synergistically regulate T-cell function to promote tumoral immune escape. Cancer Res. 2012;72(4):917-927.

36. Matsuzaki J, et al. Tumor-infiltrating NY-ESO-1 specific $\mathrm{CD}^{+} \mathrm{T}$ cells are negatively regulated by LAG-3 and PD-1 in human ovarian cancer. Proc Natl Acad Sci U S A . 2010;107(17):7875-7880.

37. Ascierto PA, et al. Initial efficacy of anti-lymphocyte activation gene-3 (anti-LAG-3; BMS986016) in combination with nivolumab (nivo) in pts with melanoma (MEL) previously treated with anti-PD-1/PD-L1 therapy. J Clin Oncol. 2017;35(15_suppl):9520.

38. Khalil DN, Smith EL, Brentjens RJ, Wolchok JD. The future of cancer treatment: immunomodulation, CARs and combination immunotherapy. Nat Rev Clin Oncol. 2016;13(5):273-290.

39. Brignone C, Escudier B, Grygar C, Marcu M, Triebel F. A phase I pharmacokinetic and biological correlative study of IMP321, a novel MHC class II agonist, in patients with advanced renal cell carcinoma. Clin Cancer Res. 2009;15(19):6225-6231.

40. Anderson AC, Joller N, Kuchroo VK. Lag-3, Tim-3, and TIGIT: co-inhibitory receptors with specialized functions in immune regulation. Immunity. 2016;44(5):989-1004.

41. Zhu C, et al. The Tim-3 ligand galectin-9 negatively regulates $\mathrm{T}$ helper type 1 immunity. Nat Immunol. 2005;6(12):1245-1252.

42. Takahashi H, Okai Y, Paxton RJ, Hefta LJ, Shively JE. Differential regulation of carcinoembryonic antigen and biliary glycoprotein by gammainterferon. Cancer Res. 1993;53(7):1612-1619.

43. Sabatos CA, et al. Interaction of Tim-3 and Tim-3 ligand regulates $\mathrm{T}$ helper type 1 responses and induction of peripheral tolerance. Nat Immunol.
2003;4(11):1102-1110.

44. Anderson AC, et al. Promotion of tissue inflammation by the immune receptor Tim-3 expressed on innate immune cells. Science. 2007;318(5853):1141-1143.

45. Jin HT, et al. Cooperation of Tim-3 and PD- 1 in CD8 T-cell exhaustion during chronic viral infection. Proc Natl Acad Sci U S A. 2010;107(33):14733-14738.

46. Sakuishi K, Apetoh L, Sullivan JM, Blazar BR, Kuchroo VK, Anderson AC. Targeting Tim-3 and PD-1 pathways to reverse T cell exhaustion and restore anti-tumor immunity. J Exp Med. 2010;207(10):2187-2194.

47. Ngiow SF, von Scheidt B, Akiba H, Yagita H, Teng MW, Smyth MJ. Anti-TIM3 antibody promotes T cell IFN- $\gamma$-mediated antitumor immunity and suppresses established tumors. Cancer Res. 2011;71(10):3540-3551.

48. Fourcade J, et al. Upregulation of Tim-3 and PD-1 expression is associated with tumor antigenspecific $\mathrm{CD}^{+} \mathrm{T}$ cell dysfunction in melanoma patients. J Exp Med. 2010;207(10):2175-2186.

49. Yan J, Zhang Y, Zhang JP, Liang J, Li L, Zheng L. Tim-3 expression defines regulatory T cells in human tumors. PLoS One. 2013;8(3):e58006.

50. Koyama S, et al. Adaptive resistance to therapeutic PD-1 blockade is associated with upregulation of alternative immune checkpoints. Nat Commun. 2016;7:10501.

51. Levin SD, et al. Vstm 3 is a member of the CD28 family and an important modulator of T-cell function. Eur J Immunol. 2011;41(4):902-915.

52. Lozano E, Dominguez-Villar M, Kuchroo V, Hafler DA. The TIGIT/CD226 axis regulates human T cell function. JImmunol. 2012;188(8):3869-3875.

53. Kurtulus S, et al. TIGIT predominantly regulates the immune response via regulatory $\mathrm{T}$ cells. J Clin Invest. 2015;125(11):4053-4062.

54. Johnston RJ, et al. The immunoreceptor TIGIT regulates antitumor and antiviral CD8(+) T cell effector function. Cancer Cell. 2014;26(6):923-937.

55. Chauvin JM, et al. TIGIT and PD-1 impair tumor antigen-specific $\mathrm{CD}^{+} \mathrm{T}$ cells in melanoma patients. J Clin Invest. 2015;125(5):2046-2058.

56. Lines JL, et al. VISTA is an immune checkpoint molecule for human T cells. Cancer Res. 2014;74(7):1924-1932.

57. Wang L, et al. VISTA, a novel mouse Ig superfamily ligand that negatively regulates $\mathrm{T}$ cell responses. JExp Med. 2011;208(3):577-592.

58. Le Mercier I, et al. VISTA regulates the development of protective antitumor immunity. Cancer Res. 2014;74(7):1933-1944.

59. Lines JL, Sempere LF, Broughton T, Wang L, Noelle R. VISTA is a novel broad-spectrum negative checkpoint regulator for cancer immunotherapy. Cancer Immunol Res. 2014;2(6):510-517.

60. Wang L, et al. Disruption of the immune-checkpoint VISTA gene imparts a proinflammatory phenotype with predisposition to the development of autoimmunity. Proc Natl Acad Sci US A. 2014;111(41):14846-14851.

61. Gao J, et al. VISTA is an inhibitory immune checkpoint that is increased after ipilimumab therapy in patients with prostate cancer. Nat Med. 2017;23(5):551-555.

62. Black CM, Armstrong TD, Jaffee EM. Apoptosis- 
regulated low-avidity cancer-specific CD8(+) T cells can be rescued to eliminate HER2/ neu-expressing tumors by costimulatory agonists in tolerized mice. Cancer Immunol Res. 2014;2(4):307-319.

63. Bulliard Y, Jolicoeur R, Zhang J, Dranoff G, Wilson NS, Brogdon JL. OX40 engagement depletes intratumoral Tregs via activating $\mathrm{Fc} \gamma \mathrm{Rs}$, leading to antitumor efficacy. Immunol Cell Biol. 2014;92(6):475-480.

64. Hirschhorn-Cymerman D, et al. OX40 engagement and chemotherapy combination provides potent antitumor immunity with concomitant regulatory $\mathrm{T}$ cell apoptosis. JExp Med. 2009;206(5):1103-1116.

65. Schaer DA, Hirschhorn-Cymerman D, Wolchok JD. Targeting tumor-necrosis factor receptor pathways for tumor immunotherapy. J Immunother Cancer. 2014;2:7.

66. Gramaglia I, Jember A, Pippig SD, Weinberg AD, Killeen N, Croft M. The OX4O costimulatory receptor determines the development of $\mathrm{CD} 4$ memory by regulating primary clonal expansion. Jimmunol. 2000;165(6):3043-3050.

67. Fraietta JA, et al. Determinants of response and resistance to $\mathrm{CD} 19$ chimeric antigen receptor (CAR) T cell therapy of chronic lymphocytic leukemia. Nat Med. 2018;24(5):563-571.

68. Chen AI, et al. Ox40-ligand has a critical costimulatory role in dendritic cell: $\mathrm{T}$ cell interactions. Immunity. 1999;11(6):689-698.

69. Ohshima Y, Tanaka Y, Tozawa H, Takahashi Y, Maliszewski C, Delespesse G. Expression and function of OX40 ligand on human dendritic cells. J Immunol. 1997;159(8):3838-3848.

70. Pan PY, Zang Y, Weber K, Meseck ML, Chen SH. OX40 ligation enhances primary and memory cytotoxic T lymphocyte responses in an immunotherapy for hepatic colon metastases. Mol Ther. 2002;6(4):528-536.

71. Adler AJ, Vella AT. Betting on improved cancer immunotherapy by doubling down on CD134 and CD137 co-stimulation. Oncoimmunology. 2013;2(1):e22837.

72. Guo Z, et al. Combined TIM-3 blockade and CD137 activation affords the long-term protection in a murine model of ovarian cancer. J Transl Med. 2013;11:215.

73. Guo Z, Wang X, Cheng D, Xia Z, Luan M, Zhang S. PD- 1 blockade and OX40 triggering synergistically protects against tumor growth in a murine model of ovarian cancer. PLoS One. 2014;9(2):e89350.

74. Curti BD, et al. OX40 is a potent immunestimulating target in late-stage cancer patients. Cancer Res. 2013;73(24):7189-7198.

75. Infante JR, et al. A phase Ib dose escalation study of the OX40 agonist MOXR0916 and the PD-L1 inhibitor atezolizumab in patients with advanced solid tumors. JClin Oncol. 2016;34(15_suppl):101.

76. Tone $\mathrm{M}$, et al. Mouse glucocorticoid-induced tumor necrosis factor receptor ligand is costimulatory for T cells. Proc Natl Acad Sci U S A. 2003;100(25):15059-15064.

77. Kanamaru F, et al. Costimulation via glucocorticoid-induced TNF receptor in both conventional and $\mathrm{CD} 25^{+}$regulatory $\mathrm{CD} 4^{+} \mathrm{T}$ cells. J Immunol. 2004;172(12):7306-7314.
78. Ronchetti S, Nocentini G, Bianchini R, Krausz LT, Migliorati G, Riccardi C. Glucocorticoid-induced TNFR-related protein lowers the threshold of CD28 costimulation in CD $8^{+} \mathrm{T}$ cells. J Immunol. 2007;179(9):5916-5926.

79. Cohen AD, et al. Agonist anti-GITR monoclonal antibody induces melanoma tumor immunity in mice by altering regulatory $\mathrm{T}$ cell stability and intra-tumor accumulation. PLoS One. 2010;5(5):e10436.

80. Lu L, Xu X, Zhang B, Zhang R, Ji H, Wang X. Combined PD-1 blockade and GITR triggering induce a potent antitumor immunity in murine cancer models and synergizes with chemotherapeutic drugs. J Transl Med. 2014;12:36.

81. Mitsui J, et al. Two distinct mechanisms of augmented antitumor activity by modulation of immunostimulatory/inhibitory signals. Clin Cancer Res. 2010;16(10):2781-2791.

82. Mahoney KM, Rennert PD, Freeman GJ. Combination cancer immunotherapy and new immunomodulatory targets. Nat Rev Drug Discov. 2015;14(8):561-584.

83. Lee HW, Park SJ, Choi BK, Kim HH, Nam KO, Kwon BS. 4-1BB promotes the survival of CD8+ T lymphocytes by increasing expression of Bcl-xL and Bfl-1. J Immunol. 2002;169(9):4882-4888.

84. Shuford WW, et al. 4-1BB costimulatory signals preferentially induce $\mathrm{CD} 8+\mathrm{T}$ cell proliferation and lead to the amplification in vivo of cytotoxic T cell responses. J Exp Med. 1997;186(1):47-55.

85. Ascierto PA, Simeone E, Sznol M, Fu YX, Melero I. Clinical experiences with anti-CD137 and anti-PD1 therapeutic antibodies. Semin Oncol. 2010;37(5):508-516.

86. Wei $\mathrm{H}$, et al. Combinatorial PD-1 blockade and CD137 activation has therapeutic efficacy in murine cancer models and synergizes with cisplatin. PLoS One. 2013;8(12):e84927.

87. Segal NH, et al. Results from an integrated safety analysis of Urelumab, an agonist antiCD137 monoclonal antibody. Clin Cancer Res. 2017;23(8):1929-1936.

88. van Kooten C, Banchereau J. CD40-CD40 ligand. J Leukoc Biol. 2000;67(1):2-17.

89. Bennett SR, Carbone FR, Karamalis F, Flavell RA, Miller JF, Heath WR. Help for cytotoxic-T-cell responses is mediated by $\mathrm{CD} 40$ signalling. Nature. 1998;393(6684):478-480.

90. Schoenberger SP, Toes RE, van der Voort EI, Offringa R, Melief CJ. T-cell help for cytotoxic T lymphocytes is mediated by CD40-CD40L interactions. Nature. 1998;393(6684):480-483.

91. van Essen D, Kikutani H, Gray D. CD40 ligand-transduced co-stimulation of T cells in the development of helper function. Nature. 1995;378(6557):620-623.

92. Vonderheide RH, Glennie MJ. Agonistic CD40 antibodies and cancer therapy. Clin Cancer Res. 2013;19(5):1035-1043.

93. Beatty GL, et al. CD40 agonists alter tumor stroma and show efficacy against pancreatic carcinoma in mice and humans. Science. 2011;331(6024):1612-1616.

94. Byrne KT, Vonderheide RH. CD40 stimulation obviates innate sensors and drives T cell immunity in cancer. Cell Rep. 2016;15(12):2719-2732.

95. Le DT, et al. Evaluation of ipilimumab in com- bination with allogeneic pancreatic tumor cells transfected with a GM-CSF gene in previously treated pancreatic cancer. JImmunother. 2013;36(7):382-389.

96. Ott PA, et al. An immunogenic personal neoantigen vaccine for patients with melanoma. Nature. 2017;547(7662):217-221.

97. Sahin U, et al. Personalized RNA mutanome vaccines mobilize poly-specific therapeutic immunity against cancer. Nature. 2017;547(7662):222-226.

98. Soares KC, et al. PD-1/PD-L1 blockade together with vaccine therapy facilitates effector $\mathrm{T}$-cell infiltration into pancreatic tumors. J Immunother. 2015;38(1):1-11.

99. Andtbacka RH, et al. Talimogene laherparepvec improves durable response rate in patients with advanced melanoma. JClin Oncol. 2015;33(25):2780-2788.

100.Puzanov I, et al. Talimogene laherparepvec in combination with ipilimumab in previously untreated, unresectable stage IIIB-IV melanoma. JClin Oncol. 2016;34(22):2619-2626.

101. Hannani D, Sistigu A, Kepp O, Galluzzi L, Kroemer G, Zitvogel L. Prerequisites for the antitumor vaccine-like effect of chemotherapy and radiotherapy. Cancer J. 2011;17(5):351-358.

102. Postow MA, et al. Immunologic correlates of the abscopal effect in a patient with melanoma. N Engl JMed. 2012;366(10):925-931.

103. Twyman-Saint Victor $C$, et al. Radiation and dual checkpoint blockade activate non-redundant immune mechanisms in cancer. Nature. 2015;520(7547):373-377.

104. Langer CJ, et al. Carboplatin and pemetrexed with or without pembrolizumab for advanced, non-squamous non-small-cell lung cancer: a randomised, phase 2 cohort of the open-label KEYNOTE-021 study.Lancet Oncol. 2016;17(11):1497-1508.

105. Sevko A, et al. Antitumor effect of paclitaxel is mediated by inhibition of myeloid-derived suppressor cells and chronic inflammation in the spontaneous melanoma model. J Immunol. 2013;190(5):2464-2471.

106.Vincent J, et al. 5-Fluorouracil selectively kills tumor-associated myeloid-derived suppressor cells resulting in enhanced T celldependent antitumor immunity. Cancer Res. 2010;70(8):3052-3061.

107. Zhu Y, et al. CSF1/CSF1R blockade reprograms tumor-infiltrating macrophages and improves response to T-cell checkpoint immunotherapy in pancreatic cancer models. Cancer Res. 2014;74(18):5057-5069.

108. Hainsworth JD, et al. A randomized, open-label phase 2 study of the CXCR4 inhibitor LY2510924 in combination with sunitinib versus sunitinib alone in patients with metastatic renal cell carcinoma (RCC). Target Oncol. 2016;11(5):643-653.

109. Yang L, et al. Expansion of myeloid immune suppressor $\mathrm{Gr}+\mathrm{CD} 11 \mathrm{~b}+$ cells in tumor-bearing host directly promotes tumor angiogenesis. Cancer Cell. 2004;6(4):409-421.

110. Yang L, et al. Abrogation of TGF beta signaling in mammary carcinomas recruits $\mathrm{Gr}-1^{+} \mathrm{CD} 11 \mathrm{~b}^{+}$ myeloid cells that promote metastasis. Cancer Cell. 2008;13(1):23-35.

111. Meyer C, et al. Frequencies of circulating MDSC correlate with clinical outcome of melanoma 
patients treated with ipilimumab. Cancer Immunol Immunother. 2014;63(3):247-257.

112. Orillion A, et al. Entinostat neutralizes myeloid-derived suppressor cells and enhances the antitumor effect of PD-1 inhibition in murine models of lung and renal cell carcinoma. Clin Cancer Res. 2017;23(17):5187-5201.

113. Johnson ML, et al. ENCORE 601: A phase II study of entinostat (ENT) in combination with pembrolizumab (PEMBRO) in patients with melanoma. JClin Oncol. 2017;35(15_suppl):601.

114. Hanks BA, Holtzhausea A, Evans K, Heid M,
Blobe GC. Combinatorial TGF- $\beta$ signaling blockade anti-CTLA-4 antibody immunotherapy in a murine $\mathrm{BRAF}^{\mathrm{V} 600 \mathrm{E}}-\mathrm{PTEN}^{-/}$transgenic model of melanoma. JClin Oncol.2014;35(15_suppl):3011.

115. Soares KC, et al. TGF- $\beta$ blockade depletes T regulatory cells from metastatic pancreatic tumors in a vaccine dependent manner. Oncotarget. 2015;6(40):43005-43015.

116. Munn DH, Shafizadeh E, Attwood JT, Bondarev I, Pashine A, Mellor AL. Inhibition of T cell proliferation by macrophage tryptophan catabolism. JExp Med.1999;189(9):1363-1372.
117. Sharma MD, et al. Plasmacytoid dendritic cells from mouse tumor-draining lymph nodes directly activate mature Tregs via indoleamine 2,3-dioxygenase. JClin Invest. 2007;117(9):2570-2582.

118. Taylor MW, Feng GS. Relationship between interferon-gamma, indoleamine 2,3-dioxygenase, and tryptophan catabolism. FASEB J. 1991;5(11):2516-2522.

119. Hellmann MD, et al. Genomic features of response to combination immunotherapy in patients with advanced non-small-cell lung cancer. Cancer Cell. 2018;33(5):843-852.e4. 